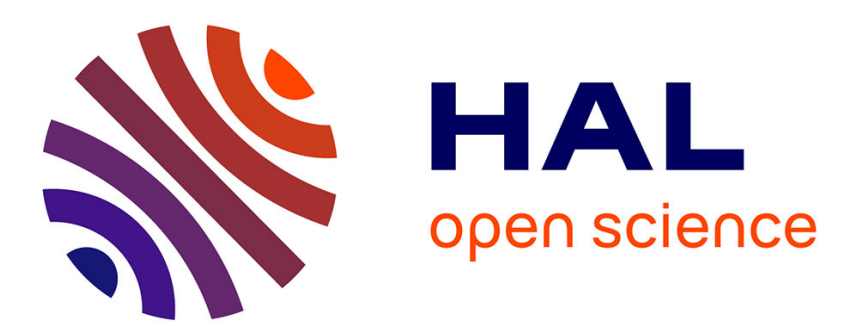

\title{
Ordre et inquiétude du discours dans le discours littéraire contemporain: Portrait de l'écrivain en animal domestique de Lydie Salvayre
}

Stéphane Bikialo

\section{- To cite this version:}

Stéphane Bikialo. Ordre et inquiétude du discours dans le discours littéraire contemporain : Portrait de l'écrivain en animal domestique de Lydie Salvayre. A. Kieliszczyk et E. Pachocinska. L'Analyse du discours: de la théorie à la pratique, Wydawnictwa Uniwersytetu Warszawskiego, p. 13-28, 2014, 978-83-235-1756-6. hal-02509324

\section{HAL Id: hal-02509324 \\ https://hal.science/hal-02509324}

Submitted on 16 Mar 2020

HAL is a multi-disciplinary open access archive for the deposit and dissemination of scientific research documents, whether they are published or not. The documents may come from teaching and research institutions in France or abroad, or from public or private research centers.
L'archive ouverte pluridisciplinaire HAL, est destinée au dépôt et à la diffusion de documents scientifiques de niveau recherche, publiés ou non, émanant des établissements d'enseignement et de recherche français ou étrangers, des laboratoires publics ou privés. 
BIKIALO Stéphane

stephane.bikialo@univ-poitiers.fr

Université de Poitiers

Titre en français : Ordre et inquiétude du discours dans le discours littéraire contemporain : Portrait de l'écrivain en animal domestique de Lydie Salvayre.

Titre en anglais : The order and anxiety of discourse in contemporary literature : Portrait de l'écrivain en animal domestique from Lydie Salvayre

Résumé en français : Cet article propose une analyse du début de L'Ordre du discours de Michel Foucault et de Portrait de l'écrivain en animal domestique de Lydie Salvayre, afin de montrer comment se théorise et s'écrit l'opposition entre ordre et inquiétude du discours dans la rencontre du discours économique et du discours littéraire. L'ouvrage de Lydie Salvayre met en effet en scène un discours économique d'autorité qui, tout en relevant de l'ordre du discours, génère variations et hétérogénéités énonciatives au sein du discours littéraire, aspects qui ressortissent de l'inquiétude du discours.

Résumé en anglais : This article analyses the beginning of Michel Foucault's The Order of Discourse along with Lydie Salvayre's Portrait de l'écrivain en animal domestique, in order to show how the opposition between order and anxiety of discourse is theorized and written at the intersection of literary and economic discourse. Lydie Salvayre's book displays an authoritative economic discourse which pertains to what Foucault calls the order of discourse, but her literary work generates variations which induce anxiety of discourse.

Mots-clés en français : interdiscours, Foucault, Salvayre, discours littéraire, analyse critique des discours

Mots-clés en anglais : interdiscourse, Foucault, Salvayre, literary discourse, Critical analysis of discourse 
Au sein des ${ }^{1}$ analyses du discours, l'analyse du discours littéraire reste assez peu représentée, ou plutôt assez peu revendiquée comme telle, en dépit des travaux de D. Maingueneau, depuis Le Contexte de l'œuvre littéraire (1993), puis dans Le Discours littéraire. Paratopie et scène d'énonciation (2004) et le collectif issu du colloque de Cerisy, L'Analyse du discours dans les études littéraires (2002) dirigé avec R. Amossy. Récemment encore (2008), D. Maingueneau s'interrogeait sur les apports respectifs de l'analyse du discours et des études littéraires soulignant que

[...] la question de la nature des relations entre analyse du discours et littérature se pose avec une acuité particulière dès lors que la plupart des spécialistes de littérature jugent tout à la fois illégitime et inefficient le recours à des problématiques d'analyse du discours dans leur domaine. [...] Les réticences des littéraires rejoignent ici celles des analystes du discours à l'égard de la littérature. [...]. Au moment où l'analyse du discours est apparue, dans les années soixante, en matière d'étude de textes il existait une sorte de répartition tacite du travail : les facultés de lettres analysaient les textes prestigieux, en prêtant une attention particulière au «style », et donc aux ressources linguistiques mobilisées par l'écrivain ; de leur côté, les départements de sciences humaines ou sociales avaient affaire à des textes de faible prestige, des «documents » qui n'étaient pas considérés comme passibles d'une approche stylistique et n'étaient étudiés que parce qu'ils donnaient accès à des réalités extralinguistiques. L'analyse du discours [...] a converti le regard sur ces « documents » : ils ont bénéficié désormais d'une attention comparable à celle des textes qui étaient étudiés dans les facultés de lettres, mais dans un cadre théorique et méthodologique très différent. Pendant plus de deux décennies cette situation a perduré tant bien que mal : l'analyse du discours a soigneusement évité les textes prestigieux, tandis que la stylistique littéraire a intégré certains outils empruntés aux courants énonciatifs et pragmatiques, mais sans pour autant mettre en cause ses gestes essentiels et les partages institutionnels qu'ils impliquent (2008:2-3).

Avec la sémiotisation du style proposée par G. Genette dans Fiction et diction (1991) reprenant l'attention inaugurée par la «stylistique» de Ch. Bally aux textes non littéraires, avec le développement des travaux de la « sociocritique » dans les années 1970-1980 mise en relation par R. Amossy $(2003,2005)$ avec l'analyse des discours, avec aussi le passage de la linguistique textuelle à l'analyse textuelle des discours dans les travaux de J.-M. Adam², les outils théoriques et méthodologiques permettent toutefois un rapprochement qui existait dès l'origine de l'analyse du discours, notamment à travers les travaux de M. Foucault.

Cela est d'autant plus manifeste et aisé lorsqu'on travaille sur la littérature contemporaine qui redonne au «document » un statut mis à distance - par la critique parfois plus que par les

\footnotetext{
1 Au pluriel, comme le proposait D. Maingueneau en titre et en présentation du numéro 117 de Langages consacré précisément aux «analyses du discours en France », où il souligne un «émiettement des modes d'approche » (p. 5) mais qui tient à son objet même, le discours, qui «désigne moins un objet empirique qu'un certain mode d'appréhension du langage » (p. 6).

${ }^{2}$ Voir ainsi l'évolution théorique amorcée dans Linguistique textuelle : des genres de discours aux textes, Nathan-Université, 1999, puis développée dans La linguistique textuelle. Introduction à l'analyse textuelle des discours, A. Colin, 2005 et dans ses rééditions successives de 2008 et 2011. Dans Le Texte littéraire. Pour une approche interdisciplinaire, Bruylant-Acadamia, 2009, J.-M. Adam et U. Heidman écrivent : « Nous pensons, comme Henri Meschonnic, que le la littérature 'se fait dans l'ordre du discours et requiert les concepts du discours'» (p. 9).
} 
auteurs - pendant quelques décennies. On a ainsi récemment pu parler de «sociologisation de la littérature » (Viart, 2011) ou de «narrations documentaires» (Ruffel, 2012). Ce mouvement ancre le discours littéraire dans l'analyse du discours, les textes apparaissant comme des «documents »; mais ce rapport au document permet également une réflexion parfois thématisée et exemplifiée dans ces discours littéraires ${ }^{3}$ - sur la spécificité du discours littéraire par rapport aux discours économique, politique, médiatique, etc.

On a choisi ici d'interroger les rapports entre discours économique et discours littéraire, au sein d'un roman qui les thématise sur le mode de la confrontation : Portrait de l'écrivain en animal domestique de Lydie Salvayre (Seuil, 2007). Tout se passe comme si ce texte s'interrogeait sur le rapport entre «l'ordre du discours » et «l'inquiétude du discours » mis en avant par Michel Foucault au début de L'Ordre du discours (1971) qu'on relira avant de montrer ses enjeux dans l'ouvrage de L. Salvayre.

\section{Ordre et inquiétude du discours}

Au début de L'Ordre du discours, M. Foucault opère une distinction théorique, et exemplifiée stylistiquement, entre « ordre » et « inquiétude » du discours :

Dans le discours qu'aujourd'hui je dois tenir, et dans ceux qu'il me faudra tenir ici, pendant des années peut-être, j'aurais voulu pouvoir me glisser subrepticement. Plutôt que de prendre la parole, j'aurais voulu être enveloppé par elle, et porté bien au-delà de tout commencement possible. J'aurais aimé m'apercevoir qu'au moment de parler une voix sans nom me précédait depuis longtemps : il m'aurait suffi alors d'enchainer, de poursuivre la phrase, de me loger, sans qu'on y prenne bien garde, dans ses interstices, comme si elle m'avait fait signe en se tenant, un instant, en suspens $[\ldots]$; et au lieu d'être celui dont vient le discours, je serais plutôt au hasard de son déroulement, une mince lacune, le point de sa disparition possible (p. 7-8).

Aux expressions «tenir un discours », «prendre la parole », qui disent la maîtrise d'un sujet, Foucault oppose «se glisser subrepticement», «être enveloppé par la parole », «se loger dans les interstices » d'une parole déjà-là... Le locuteur serait ainsi moins celui « dont vient le discours » qu'une «mince lacune, le point de sa disparition possible», moins un sujet « origine », maître de son langage qu'un sujet « effet » pour reprendre la distinction proposée par J. Authier-Revuz :

$\mathrm{Si}$, en effet, on s'appuie à un sujet « origine », A, celui de la psychologie et de ses variantes «neuronales » ou sociales, source intentionnelle du sens qu'il exprime à travers une langue instrument de communication - ce qui est le cas de façon générale dans les approches pragmaticocommunicationnelles -, il est alors cohérent de considérer que l'énonciateur est en mesure de (se) représenter son énonciation et le sens qu'il y « produit », et qui peut lui être transparent [...].

$\mathrm{Si}$, au contraire, on s'appuie à un sujet «effet», B, celui assujetti à l'inconscient de la psychanalyse, dépossédé de la maîtrise de son dire [...], on considèrera que le dire ne saurait être

\footnotetext{
${ }^{3}$ Voir Bikialo, 2010, 2011 et 2013 pour ces enjeux évoqués au sujet d'autres textes et d'autres problématiques.
} 
transparent à l'énonciateur, auquel il échappe, irreprésentable, déterminé qu'il est par l'inconscient (et l'interdiscours). (1994:17)

L'ouverture de L'Ordre du discours s'écrit au conditionnel passé («j'aurais voulu», «j'aurais aimé », « il m'aurait suffi »...), à valeur d'irréel du passé : il s'agit donc bien d'un discours intenable, qui n'a pu être tenu: ce conditionnel est donc une première exemplification de «l'ordre du discours » (le sujet tient sa position de maitrise) et en même temps de «l'inquiétude du discours » (le sujet ne fait pas ce qu'il aurait voulu faire); le texte continue ensuite d'exemplifier cette absence de maîtrise :

J'aurais aimé qu'il y ait derrière moi (ayant pris depuis bien longtemps la parole, doublant à l'avance tout ce que je vais dire) une voix qui parlerait ainsi : «Il faut continuer, je ne peux pas continuer, il faut continuer, il faut dire des mots tant qu'il y en a, il faut les dire jusqu'à ce qu'ils me trouvent, jusqu'à ce qu'ils me disent - étrange peine, étrange faute, il faut continuer, c'est peutêtre déjà fait, ils m'ont peut-être déjà dit, ils m'ont peut-être porté jusqu'au seuil de mon histoire, devant la porte qui s'ouvre sur mon histoire, ça m’étonnerait si elle s'ouvre. » (p. 8)

Ce qui détermine le sujet « effet » est ici envisagé comme issu d'une «voix » de « derrière », citée (entre guillemets) sans que la référence soit précisée, sans que la source soit explicitement identifiée, nommée ; cette voix, c'est celle de la fin de L'Innommable de Samuel Beckett ${ }^{4}$, dans un texte qui souligne l'absence de maîtrise sur le langage, où les mots sont en position de sujet (syntaxique et sémantique) et le locuteur en position de complément : «jusqu'à ce qu'ils me disent $»^{5}$. L'intertextualité et l'interdiscours apparaissent ici pour souligner que «ça parle toujours avant et ailleurs » (Pêcheux, 1990), que les mots sont toujours déjà « habités » ${ }^{6}$, que l'enjeu du discours est dans ce rapport à autrui.

Cet interdiscours est par ailleurs mis en œuvre à travers une prosopopée du désir et de l'institution, incarnations respectives de l'inquiétude et de l'ordre du discours :

Le désir dit : «je ne voudrais pas avoir à entrer moi-même dans cet ordre hasardeux du discours ; je ne voudrais pas avoir affaire à lui dans ce qu'il a de tranchant et de décisif ; je voudrais qu'il soit tout autour de moi, comme une transparence calme, profonde, indéfiniment ouverte, où les autres répondraient à mon attente, et d'où les vérités, une à une, se lèveraient; je n'aurais qu'à me laisser porter, en lui, et par lui, comme une épave heureuse. » Et l'institution répond : « Tu n'as pas à craindre de commencer; nous sommes tous là pour te montrer que le discours est dans l'ordre des lois ; qu'on veille depuis longtemps sur son apparition, qu'une place lui a été faite, qui

\footnotetext{
${ }^{4}$ S. Beckett, L'Innommable, éditions de Minuit, 1953, p. 213.

${ }^{5}$ A la fin de la conférence, Foucault identifiera un référent possible de cette voix de derrière en la personne de Jean Hyppolite, auquel il succède, dans un bel effet de boucle : «Je sais bien maintenant quelle est la voix dont j'aurais voulu qu'elle me précède, qu'elle me porte, qu'elle m'invite à parler et qu'elle se loge dans mon propre discours. Je sais ce qu'il y avait de redoutable à prendre la parole, puisque je la prenais en ce lieu d'où je l'ai écouté, et où il n'est plus, lui, pour m'entendre. » (M. Foucault, op. cit. , p. 81-82) : par cette clôture rendant hommage à son prédécesseur, M. Foucault à la fois répond à l'injonction institutionnelle, à la dimension rituelle de «l'ordre du discours » qu'il a identifiée précédemment, et met en avant cette dépossession du discours, qui est un des signes du discours inquiet.

${ }^{6}$ « Aucun membre de la communauté verbale ne trouve jamais des mots de la langue qui soient neutres, exempts des aspirations et des évaluations d'autrui, inhabités par la voix d'autrui. Non, il reçoit le mot par la voix d'autrui, et ce mot en reste rempli [...]. Sa propre intention trouve un mot déjà habité. »: M. Bakhtine, cité par T. Todorov dans M. Bakhtine, le cercle dialogique, Seuil, 1981, p. 77.
} 
l'honore mais le désarme ; et que, s'il lui arrive d'avoir quelque pouvoir, c'est bien de nous, et de nous seulement, qu'il le tient. » (p. 9)

Ces prosopopées sont une manière de mettre en scène le «ça parle », le ça désignant ici ce qu'il y a en nous de profond (le désir) mais aussi les contraintes sociales (l'institution) telles qu'elles se manifestent dans le discours à travers les «genres de discours », «facteurs de régulation de la créativité langagière $»^{7}$. Mais comme l'indique Foucault, si le désir est naturellement du côté de l'inquiétude, il se peut aussi que l'institution le soit aussi, que l'ordre du discours porte en lui cette inquiétude d'un danger :

Mais peut-être cette institution et ce désir ne sont-ils pas autre chose que deux répliques opposées à une même inquiétude : inquiétude à l'égard de ce qu'est le discours dans sa réalité matérielle de chose prononcée ou écrite ; inquiétude à l'égard de cette existence transitoire vouée à s'effacer sans doute, mais selon une durée qui ne nous appartient pas ; inquiétude à sentir sous cette activité, pourtant quotidienne et grise, des pouvoirs et des dangers qu'on imagine mal ; [...].

Mais qui y a-t-il donc de si périlleux dans le fait que les gens parlent, et que leurs discours indéfiniment prolifèrent ? Où est donc le danger ? (p. 9-10)

A la phrase comme «événement évanouissant» de Benveniste ${ }^{8}$ répond ici «l'existence transitoire » du discours ; le discours littéraire relève à la fois de l'ordre de l'institution ${ }^{9}$ et du désir : il est «inquiétude du discours ». L'inquiétude, nous disent des voix antérieures ${ }^{10}$, c'est l'absence de repos (du corps et de l'esprit), le mouvement; l'inquiétude démange mais stimule, alors que l'ordre exclut, classe, interdit, ritualise à travers des «procédures de contrôle » :

Je suppose que dans toute société la production du discours est à la fois contrôlée, sélectionnée, organisée et redistribuée par un certain nombre de procédures qui ont pour rôle d'en conjurer les pouvoirs et les dangers, d'en maîtriser l'événement aléatoire, d'en esquiver la lourde, la redoutable matérialité (p. 10-11)

L'analyse du discours, qui s'intéresse habituellement davantage à ce qui met de l'ordre dans le discours, permet de penser le discours littéraire comme discours inquiet, où le sujet est plus effet que maître, origine de son discours, où la conception anthropologique (l'énonciation, l'écriture comme construction de soi) l'emporte sur la conception pragmatique (l'écriture comme action sur autrui), et où le discours vise non pas à apporter des réponses mais à

\footnotetext{
${ }^{7}$ M. Bakhtine, Esthétique de la création verbale [1952-1953], Gallimard, 1984 : « Le locuteur reçoit donc, [...] les formes non moins prescriptives pour lui de l'énoncé, c'est-à-dire les genres du discours » (p. 287).

${ }^{8}$ E. Benveniste, Problèmes de linguistique générale, t. 2, Gallimard, 1974, p. 227.

${ }^{9}$ M. Foucault évoque à plusieurs reprises «l'ordre du discours littéraire » (p. 29), en mentionnant notamment «l'auteur» comme «principe de raréfaction» (p. 28) du discours ainsi que les classifications (p. 23) et les sociétés de discours (p. 42).

${ }^{10}$ Voir les belles citations isolées par le $T L F$ dans l'article « Inquiétude ».
} 
inquiéter. Pour Christian Prigent, c’est la poésie qui manifeste le plus cette «inquiétude du discours $\gg$ :

Comme tout monde humain, mais plus qu'aucun autre peut-être, notre monde est un monde en manque de sens. La demande de sens y est donc d'autant plus acharnée. L'actualité en offre de nombreux symptômes. [...] Dans un tel contexte, on peut comprendre que nos contemporains manifestent peu de goût pour ce qui n'apporte aucune apaisante clarté ni aucun savoir stabilisé. Rien de vraiment étonnant dans leur dédain de la littérature « difficile». Rien de mystérieux non plus ans ce que j'ai appelé ailleurs la volonté d'oublier la «modernité » en art et en littérature. J'appelle ici modernité ce qui érode l'assurance des savoirs d'époque, défait le confort formel et propose moins du sens qu'une inquiétude sur les conditions mêmes de production d'un sens communément partageable (1996: 9-10).

\section{L'ordre du discours chez Lydie Salvayre}

Dans La Médaille (Seuil, 1993) mais aussi dans Quelques conseils utiles aux jeunes huissiers (Verticales, 1997) ou «Avertissements et préconisations aux chefs d'entreprise afin de les prémunir contre le ressentiment de leurs employés » (Dire le travail, La Licorne, 2012), et enfin dans Portrait de l'écrivain en animal domestique, œuvre sur laquelle je vais me concentrer, Lydie Salvayre s'efforce, s'amuse à mettre en scène un «discours sérieux » (Hamon, 1996), un discours de l'ordre, de l'ordre du discours tel que l'expose M. Foucault. On s'appuiera sur un assez long extrait qui servira de point de départ à toutes les analyses qui suivent :

Parle à ma bourse, ma tête est malade, aimait-il à proférer. Ou encore : Trop penser nuit. Et il ne dissimulait pas ses bâillements lorsque Alain Dongue, son dircom, se lançait dans des considérations d'ordre théorique sur le concept du trickle down effect tel qu'il avait été élaboré par Ricardo et Smith, encore appelé effet de ruissellement, et, selon lequel les riches, ne pouvant dépasser un certain seuil d'accumulation des richesses, se voyaient contraints, la mort dans l'âme, de les laisser ruisseler sur les pauvres, argument auquel on pouvait objecter que le désir de s'enrichir ne rencontrait jamais de limites objectives et ce, pour la bonne raison que chez un milliardaire l'argent n'était nullement corrélé à la satisfaction de ses besoins (manger, boire, dormir et faire pipi-caca, si l'on y réfléchit, reviennent à peu de chose) et que ce trickle down effect, en dépit de son intitulé moderniste, n'était rien d'autre que la version immonde de l'absurde chimère du Paradis biblique, etc. etc., jusqu'au moment où Tobold s'exclamait : Vos grandes phrases me soûlent, Dongue, ce qui faisait dire à ses détracteurs qu'il était tout simplement borné et qu'en guise de cervelle il disposait d'un pois.

La réponse quelque peu brutale de Tobold à l'admirable suggestion de Cindy de fonder une œuvre charitable m'amena à préciser, dans l'évangile que je rédigeais à sa gloire, la manière remarquablement binaire dont fonctionnait sa pensée.

Tobold disait yes or no, pile ou face (il avait horreur de la tiédeur et, plus que tout, de la tiédeur économique), tromper ou être trompé, tuer ou être tué. Le mystère de la trinité lui demeurait parfaitement étranger. Et dès que, dans la bouche d'un autre, deux possibilités versaient dans une troisième, trouble et énigmatique, Tobold l'écrasait d'un Basta ! Ou d'un Ça va ! Ou s'il s'agissait de Cindy, d'un Ferme-la ! sans appel.

Et les choses redevenaient magiquement simples.

Plus de chicane.

L'effronté était mis au tapis.

Qu'il dégage.

Tobold, écrivis-je tout en sachant que cette phrase ne franchirait jamais la censure patronale, Tobold a l'ignorance péremptoire et un esprit d'affirmation qui aplatit toutes les subtilités. C'est là sans doute, développai-je, l'un des secrets de sa phénoménale réussite (et celui de mon phénoménal échec, ne puis-je me défendre de penser, non sans vanité, comme si l'échec était en lui-même un gage de talent littéraire, quelle stupidité !). Les spéculations financières, comme les attaques 
surprises, honnissent le méandre, l'équivocité, la tergiversation, l'entre-deux vacillant et, plus encore, l'obscurité du poème qui ne fait qu'ajouter à l'obscurité de la vie. (p. 72-73)

Selon $\mathrm{Ph}$. Hamon, le « discours sérieux » présente un certain nombre de caractéristiques :

Son rêve et son modèle sont le performatif, discours idéal de l'autorité efficiente, de transparence et d'univocité efficace [...]. Il se veut « efficace », « réaliste», et se présente comme un discours vérifiable et crédible, comme un «pacte de créance » souscrit entre un auteur et un lecteur [...] Le discours sérieux est donc un discours réaliste, autoritaire et autorisé. Il est du côté du pouvoir [...]. Les discours religieux, juridiques, scientifiques et politiques incarnent parfaitement ce discours sérieux. Ce sont tous des discours persuasifs, assertifs et argumentatifs [...], citationnels (ils se réfèrent toujours à quelque texte canonique sacré) (1996 : 59-63)

Il y a là une caractérisation assez fidèle du discours du «dircom », Alain Dongue, en particulier dans sa nature «citationnelle» et dans son rapport au pouvoir, à «l'autorité efficiente $»^{11}$.

\subsection{Un discours citationnel.}

Un des enjeux d'une partie de la littérature contemporaine est de se demander que faire de ce moment discursif qui fait du discours économique, managérial l'interdiscours majeur de notre époque, saturant le discours quotidien ${ }^{12}$. Une des réponses de L. Salvayre est de laisser place à ce que Genet appelait « la langue de l'ennemi » :

Il me semble qu'il y a deux pièges dans lesquels il ne faut pas tomber ; c'est soit être victime sans le savoir du discours publicitaire et le reproduire sans conscience, ça c'est la pire des choses qui puisse arriver, soit produire un nouveau discours publicitaire qui se substituerait ou qui viendrait prolonger le discours publicitaire en vigueur. Il me semble que s'il y a une position littéraire c'est celle qui consiste à prendre en compte la mutation du discours, l'envahissement du discours publicitaire et rendre compte de cette mutation de la langue, de cette violence que la langue subit et faire travailler la langue pour que cette invasion apparaisse dans sa violence ${ }^{13}$.

Cet envahissement du discours est d'abord sensible à travers le lexique, notamment l'abréviation « dircom » (terme répertorié comme issu du vocabulaire managérial depuis la fin des années $1980^{14}$ ), mais plus généralement un vocabulaire financier omniprésent dans le discours de Tobold :

Tobold [...] déroula par prudence (il y était rodé) des propos insipides émaillés des mots défi, volonté, conquête, conjecture, optimisation (deux fois), challenge, indice, axe fort, analyse convergente, croissance rapide (deux fois), connexion, organigramme, panel, quota, [...] (p. 21)

\footnotetext{
${ }^{11}$ Autorité toute relative, puisque l'autorité absolue se situe du côté de Tobold, qui n'a que faire du discours, se contentant d'ordres.

12 Les écrivains E. Arlix et J.-C. Massera écrivent ainsi, dans Le Guide du démocrate (Lignes, 2010) : «L'influence permanente du langage de l'économie et du marketing sur la vie du démocrate est telle que le démocrate, sans en avoir pleinement conscience, utilise au quotidien une bonne flopée de formules, d'expressions, de périphrases d'une pauvreté accablante. » (p. 56). Voir sur ce point Bikialo, 2010 et 2011.

${ }^{13}$ L. Salvayre, entretien avec Sophie Bonnet, pour « Le village des idiots », 1999.

${ }^{14}$ Marque déposée en 1987 par le mensuel L'Expression d'entreprise, datée de 1991 dans Claudette Groud et Nicole Serna, Regards sur la troncation en français contemporain, INALF, 1996.
} 
Dans cet extrait, le discours économique de Tobold est mis à distance non seulement par le statut de discours rapporté explicite marqué par des termes métalinguistiques (« des propos insipides émaillés des mots défi, volonté »; c'est le cas aussi du discours de Dongue qui reprend la théorie du «trickle down effect » issue de Ricardo et Smith. Cet exemple montre bien comment le discours littéraire intègre un discours économique, en faisant porter par un personnage ce qui apparaît comme une reformulation des critiques que Jean Ziegler, homme politique et sociologue, a pu formuler à l'égard de cette théorie dans Les Nouveaux maitres du monde et ceux qui leur résistent. On le constate en particulier dans le lien établi avec la religion par R. Sennet :

\begin{abstract}
Richard Sennet est professeur à la London School of economics. Lors d'une récente discussion à Vienne, il me dit: 'Ce fantasme du trickle down effect ne pouvait naître que dans le cerveau d'économistes aux origines judéo-chrétiennes. Il est l'exacte reproduction de l'absurde chimère du paradis de la Bible. Crevez bonnes gens du tiers-monde et d'ailleurs ! Une vie meilleure vous est promise au paradis. [...] $]^{15}$.
\end{abstract}

Cette convocation d'analogies ou de discours religieux dans le discours économique libéral est fréquent et Portrait de l'écrivain en animal domestique s'en fait le reflet : le discours de Tobold en effet saturé d'allusions ou de références religieuses, dans le nom même que Tobold donne à la bibliographie que l'auteure écrit sur lui : «l'évangile» («lequel m'avait chargé d'écriture son évangile (c'était le mot dont il avait usé mi-amusé mi-sérieux)», p. 9). Dans l'extrait, les termes trinité, mystère renvoient à cette «cette langue religieuse qu'il s'était mis en tête de parler » (p. 25). Dans ce pastiche parodique du discours économique contemporain, on peut ajouter la convocation du vocabulaire sportif qui a été identifié comme étant un des traits du discours libéral, discours de la galvanisation empruntant fréquemment son vocabulaire aux sports automobiles ou de combat: dans l'extrait: «chicane», «mis au tapis $\gg .$.

\title{
2.2. Un discours de vérité.
}

Dans les procédures de contrôle qu'il évoque, Foucault accorde une importance particulière à la « volonté de vérité », qui selon lui prend de plus en plus de place tout en étant celle dont on parle le moins dans la mesure où on a tendance à valoriser la vérité en ignorant son caractère de «machine à exclure » (1971 : 22). Il donne l'exemple de l'économie ; de fait, le discours de Tobold est un discours de vérité, d'une vérité péremptoire qui relève de ce qu'on a pu

\footnotetext{
${ }^{15}$ J. Ziegler, Les Nouveaux maitres du monde et ceux qui leur résistent, Fayard, 2002.
} 
appeler dans les sciences sociales la «raison économique ${ }^{16}$ et en analyse du discours «l'évidence du discours néo-libéral ${ }^{17}$; cela se manifeste dans "l'ignorance péremptoire »et l'«esprit d'affirmation » de Tobold, repérable par des modalités phrastiques affirmatives et injonctives («vos grandes phrases me soûlent »; «Basta!»; «Ferme-la »), mais également par les aphorismes qui inaugurent l'extrait ; ce discours du pouvoir, de «l'autorité efficiente » est récurrent dans le roman, comme en témoignent un grand nombre d'expressions métadiscursives : «il avait une singulière propension à s'exprimer par des aphorismes qu'il empruntait aux Évangiles en retournant leur sens» (p. 23) $)^{18}$, il «affectionne les sentences, devise et paraboles » (p. 48) :

Car Tobold ne souffre nulle objection.

Car Tobold jamais ne se trompe.

Car un P.-D.G. de sa trempe jamais ne se trompe et partage avec le Saint-Père le dogme de l'infaillibilité. (p. 121)

Ce «dogme de l'infaillibilité » se manifeste par une emprise de Tobold sur le discours de la narratrice. Il est celui qui commande son écriture, comme en témoigne l'omniprésente interrogation à valeur d'injonction dès l'incipit : «c'est noté ?»

J'avais le cou meurtri à cause de la laisse, et l'esprit fatigué de l'entendre me dire C'est noté ? vingt-fois par jour C'est noté ? sur le ton qu'il réservait au personnel de service C'est noté ? (p. 9)

On retrouve cette dimension «péremptoire » et «expéditive » dans les relations interlocutives (la manière dont Tobold interrompt Dongue, la «réponse quelque peu brutale » faite à Cindy). Il s'agit bien d'un discours du pouvoir qui présente en outre la particularité d'être confronté à un discours littéraire, en raison de la fiction narrative mise en place par L. Salvayre, à savoir un récit fait par une narratrice auteure écrivant la biographie de ce personnage, comme le souligne la fin de l'extrait en thématisant le mépris de Tobold :

Je suis sûr de mon fait, rétorqua-t-il, non sans sécheresse. La raison marchande [...] ne peut que triompher. Sa marche en avant est irréversible. Toute la planète d'ailleurs est convertie à son principe, la Russie est convertie à son principe, la Chine est convertie à son principe, [...] les journalistes, les juges, les professeurs, les boulangers, les cuisiniers, les garagistes sont convertis à son principe. Elle ne connait pour ennemis que trois poètes (il insista sur le è de poète avec une intention méprisante) et deux cinglés. (p. 86)

\footnotetext{
${ }^{16}$ A. Gorz, Métamorphoses du travail. Critique de la raison économique, Galilée, 1988, repris en «folio essais », 2004.

${ }^{17}$ Thierry Guilbert, $L$ '« évidence » du discours néolibéral. Analyse dans la presse écrite, éditions du Croquant, coll. «Savoir/Agir », 2011. L'auteur montre comment cette évidence s'appuie sur les notions de «naturel», d'« efficacité », de « rationalité » (p. 18-19).

${ }^{18}$ Cette inversion de sens est caractéristique du discours néolibéral selon A. Bihr, La Novlangue néolibérale. La rhétorique du fétichisme capitaliste, éditions Page deux, 2007.
} 
Le discours du pouvoir se manifeste par les modalisations épistémiques de certitude («je suis sûr de mon fait »), la présence du verbe « rétorquer » en incise, la négation exceptive qui vaut affirmation, ainsi que le déterminant totalisant «toute » qui devrait suffire mais est détaillé par une liste où le prédicat est répété à l'identique ; dans cette énumération apparaissent les effets de parallélisme et de liste fréquents dans l'ordre du discours et qu'on retrouve aussi dans les structures binaires. Le discours sérieux se manifeste en effet par un ensemble de traits relevant d'un discours, d'un rythme, d'une pensée binaires (« la manière remarquablement binaire dont fonctionnait sa pensée »). Ainsi, dans «Tobold disait yes or no, pile ou face, chaud ou froid », la conjonction utilisée ici est bien le « ou » d'alternative - qui s'oppose au « ou » d'équivalence - ce que souligne le caractère antonymique des termes coordonnés, ou, par la suite, l'alternance actif / passif («tromper ou être trompé, tuer ou être tué »). La structure binaire se retrouve aussi dans le rythme des aphorismes : «Parle à ma bourse, ma tête est malade », au rythme 4/5, avec une double proposition. Elle s'observe aussi dans le travail de mise en page, de phrases courtes avec retour à la ligne et rôle du blanc typographique comme renforcement assertif. Les personnages enfin s'opposent comme à la fin de l'extrait avec les formules symétriques et antithétiques : «sa phénoménale réussite » opposé à «mon phénoménal échec »; «l'ignorance péremptoire et un esprit d'affirmation » opposé à : «le méandre, l'équivocité, la tergiversation, l'entre-deux vacillant et, plus encore, l'obscurité du poème) ».

\section{L'inquiétude du discours}

Intégrer ce discours de l'ordre dans le cadre d'un texte littéraire, c'est certes pouvoir le mettre à distance, mais c'est plus finement lui faire jouer le rôle d'élément d'inquiétude. Cet interdiscours économique amène en effet de la variation dans l'œuvre littéraire. Or, ce que refuse ce discours de l'ordre (le discours puriste ${ }^{19}$ ou le discours médiatique, politique ${ }^{20}$ ), c'est la variation, l'hétérogénéité.

\subsection{Variations}

Dans La Variation sociale en Français, Françoise Gadet commente le caractère constitutif de l'hétérogénéité dans toute langue et reprend les principes qui structurent cette

\footnotetext{
${ }^{19}$ A. Rey le rappelle parfaitement dans L'Amour du français. Contre les puristes et autres censeurs de la langue, Points, 2009 : «A toute passion, cependant, il faut un objet simple ou, au moins reconnaissable. Le multiple, le changeant, l'imprévisible déconcertent ».

${ }^{20}$ Voir Bikialo, 2010, et en particulier les analyses du texte de Jean-Pierre Siméon, «Objection du poème », Sermons joyeux, Les Solitaires intempestifs, 2004.
} 
diversité (dimensions diachroniques, géographiques, sociales et situationnelles), et les quatre types de variation posées par Labov auxquelles elle ajoute la variation diamésique. L'écriture de L. Salvayre repose en grande partie - c'est un sinon le trait principal de son style - sur la présence de ces variations, comme en témoigne notre extrait.

Ainsi les aphorismes initiaux, qui renvoient à un discours d'autorité, relèvent-ils aussi d'une forme de jeu sur les mots, de «non-coïncidence du discours à lui-même » (Authier-Revuz, 1995-2012) : «Parle à ma bourse, ma tête est malade » reprend l'expression populaire «parle à mon cul, ma tête est malade », avec une syllepse sur «bourse » jouant sur la polysémie (sens anatomique, économique et financier). À la variation diaphasique liée aux registres de langue familiers et courants, s'ajoute la variation diastriatique : Tobold est en effet un personnage qui porte en lui une forme de variation : personnage issu d'une classe populaire, «parvenu », il mêle la langue populaire et le discours économique comme modèle absolu de ce qu'on appelle les «nouveaux riches». L'alternance entre le discours familier voire vulgaire de Tobold et les efforts et effets « littéraires » de la narratrice (« il s'en fallut de peu que je l'injuriasse », p. 131) notamment par l'emploi récurrent des subjonctifs imparfaits et du passé simple, relève de la variation diaphasique, mais également et des variations diachronique et diamésique (le subjonctif imparfait et le passé simple étant considérés comme liés à un état de langue ancien et à la langue écrite).

\subsection{Hétérogénéités énonciatives}

Portrait de l'écrivain en animal domestique est une œuvre dialogique. Le discours de la narratrice est en permanence occupé, voire assiégé, par des discours autres, de Tobold en particulier, parfois rapportés explicitement (en discours direct ou indirect), parfois intégrés à son propre discours, dans la bivocalité du discours indirect libre (Authier-Revuz, 2001 : 200201). Ainsi dans notre extrait se succèdent du DD de Tobold puis la narration qui annonce des considérations d'ordre théorique par Alain Dongue, rapportées au discours indirect libre, avec une bivocalité telle qu'on ne sait par exemple par à qui attribuer la parenthèse «(manger, boire, dormir et faire pipi-caca, si l'on y réfléchit, reviennent à peu de choses) », puisqu'il serait logique d'attribuer ce commentaire à Dongue mais que le vocabulaire familier ne lui correspond pas; puis de nouveau du DD de Tobold et enfin du DI des «détracteurs » de Tobold. Puis la narratrice se cite, dans le livre qu'elle est en train d'écrire («m'amena à préciser, dans l'évangile que je rédigeais à sa gloire ») si bien que le paragraphe suivant semble être du DD, de l'auto-citation, ce que confirment les incises «écrivis-je », « développai-je » qui suivent, renvoyant à une mise en forme écrite. Mais dans ce discours de 
la narratrice la voix de Tobold s'entend en permanence : «l'effronté était mis au tapis, qu'il dégage ». La narratrice semble de plus en plus se réfugier dans les parenthèses face à ce discours qui l'envahit, sorte d'ilot, de refuge ou de niche. Le discours de Tobold semble assimilé par la narratrice, mettant au centre de cet ouvrage la «non-coïncidence interlocutive », que J. Authier-Revuz définit comme «la rencontre par l'énonciateur dans les mots de son discours, de mots venus d'ailleurs » $(1995: 235)$.

\section{Conclusion}

Le rapport entre discours économique, néo-libéral et discours littéraire est ici thématisé comme un combat; un combat conscient de la part du discours économique puisque pour Tobold «il n'y aurait, à ses yeux, victoire plus exquise que celle remportée sur la littérature » (p. 95); un combat dont les incarnations sont les personnages de Tobold, d'une part, et la narratrice, d'autre part, respectivement allégories du discours financier et $\mathrm{du}$ discours littéraire. Ce combat semble voir la victoire du discours économique qui envahit le discours de la narratrice dans des formes de paroles autres assimilées, la narratrice ne restant pas «étanche » (p. 89) aux paroles de Tobold comme elle l'espérait mais sn discours se faisant au contraire diaphonique ${ }^{21}$.

Mais le discours littéraire, en tant que genre de discours second, englobe l'ensemble, et se sert de «la langue de l'ennemi » pour promouvoir, dans le texte, ce que ce discours économique rejette, à savoir la variation, l'hétérogénéité, menant à «l'équivocité, la tergiversation, l'entredeux vacillant $»\left(\right.$ p. 73). C'est bien un « autre versant du langage $»^{22}$, un discours inquiet qui est mis en œuvre. C'est en cela que le discours littéraire relève de l'analyse du discours, et en particulier d'une analyse de discours critique, comme le souligne un économiste, Frédéric Lordon, qui a fait le choix d'écrire un texte littéraire - une «comédie sérieuse en vers en alexandrins » - sur la crise générée par le capitalisme et les accords entre le pouvoir politique et les banques entre 2007 et 2009 :

On peut analyser la crise financière sous toutes ses coutures, raffiner l'argument autant qu'on veut, démonter les systèmes, exposer les rouages, tout ça ne vaudra jamais une image bien choisie qui fait bouillir les sangs ou, comme le dit fort à propos une expression commune, qu'on prend en

\footnotetext{
${ }^{21}$ Concept qui désigne « la reprise et l'intégration du discours de l'interlocuteur dans le discours du locuteur » selon E. Roulet \& alii, L'Articulation du discours en français contemporain, Peter Lang, 1985.

${ }^{22}$ Selon M. Aquien (1997), Le langage sur ce versant est non utilitaire, non instrumental, déborde la fonction de communication (par des bruits, des ambiguités linguistiques, des enjeux sociaux tels que rapports de courtoisie, de force, de séduction..., et au niveau de l'individu, des émergences langagières brouillant la pure information, non destinées à être communiquées), est individuel, n'émerge qu'en situation, n'est pas fait pour être compris (même de celui qui l'émet) et opère à partir du signifiant qui joue un rôle actif dans l'avancée de ce qui est dit, au même titre que le signifié.
} 
pleine gueule - la gueule : le corps. [...] Il ne faut plus seulement dire la crise capitaliste, il faut la montrer, ou bien la faire entendre. (p. 131-132)

\section{RÉFÉRENCES BIBLIOGRAPHIQUES}

Adam, J.-M. et Heidman, U., 2009, Le Texte littéraire. Pour une approche interdisciplinaire, Bruylant-Acadamia.

Amossy, R. et Maingueneau, D. (dir.), 2003, L'Analyse du discours dans les études littéraires, Toulouse, PU Mirail.

Amossy, R., 2003, «La dimension sociale du discours. L'analyse du discours et le projet sociocritique », dans L'Analyse du discours dans les études littéraires, Toulouse, PU Mirail, p. 63-74. Amossy, R., 2005, «Présentation : analyse du discours et sociocritique », Littérature n 140, p. 3-13. Aquien, M., 1997, L'Autre versant du langage, Paris, Corti.

Authier-Revuz, J., 1994, «Deux ou trois choses sur les rapports de la langue à ce qui n'est pas elle », Education Permanente $\mathrm{n}^{\circ} 120$, p. 13-20.

Authier-Revuz, J., 1995-2012, Ces mots qui ne vont pas de soi : Boucles réflexives et noncoïncidences du dire, Paris, Larousse, repris chez Lambert-Lucas, 2012.

Authier-Revuz, J., 2001, «Modalisation par discours autre et bivocalité », dans Une Langue : le français (dir. R. Tomassone), Hachette, p. 200-201.

Bikialo, S., 2010, «La maladie de la langue » dans La Langue littéraire à l'aube du XXIème siècle », PU Dijon, p. 147-160.

Bikialo, S., 2011, "J'ai un clou sur la langue". Phraséologie néo-libérale et langue littéraire au XXIème siècle », L'Information grammaticale $\mathrm{n}^{\circ} 130$, p. 41-49.

Bikialo, S., Rault, J., 2013, «La 'crise' : circulation et fiction », Epistémocritique, volume 12, Juin 2013, http://www.epistemocritique.org/spip.php?rubrique72\&lang=fr

Foucault, M., 1971, L'Ordre du discours, Paris, Gallimard.

Gadet, F., 2003, La Variation sociale en Français, Paris-Gap, Ophrys.

Hamon, Ph., 1996, L'Ironie, Paris, Hachette.

Maingueneau, D., 2008, "Analyse du discours et littérature : problèmes épistémologiques et institutionnels », Argumentation et Analyse du Discours, 1 | 2. URL : http://aad.revues.org/351

Pêcheux, M., 1990, L'Inquiétude du discours, textes choisis et présentés par D. Maldidier, Editions des Cendres, 1990.

Prigent, C., 1996, A quoi bon encore des poètes ?, Paris, POL.

Ruffel, L., 2012, «Un réalisme contemporain : les narrations documentaires », Littérature $\mathrm{n}^{\circ}$ 166, p. 13-25,

Viart, D., 2011, «Écrire le travail : Vers une sociologisation du roman contemporain?», Raison publique $\mathrm{n}^{\circ} 15$, p. 13-34. 